\title{
Chiral Imbalance in QCD and its consequences
}

\author{
Alexander Andrianov ${ }^{1,2, \star}$ Vladimir Andrianov ${ }^{1, \star \star}$ and Domenec Espriu ${ }^{2, \star \star \star}$ \\ ${ }^{1}$ Faculty of Physics, Saint Petersburg State University, Universitetskaya nab. 7/9, Saint Petersburg 199034, \\ Russia, and \\ ${ }^{2}$ Departament de Física Quàntica i Astrofísica and Institut de Ciéncies del Cosmos (ICCUB), Universitat de \\ Barcelona, Martí i Franqués 1, 08028 Barcelona, Spain
}

\begin{abstract}
Under extreme conditions of high temperature and/or large quark (baryon) density, the vacuum of QCD changes its properties, and deconfinement, chiral symmetry restoration as well as chiral symmetry breaking take place. These transitions (phases) are accompanied by the rapid change in the rate and nature of topological transitions connecting different topological sectors. The heavy ion collisions (HIC) program opens a possibility to study these phenomena in so-called non-Abelian Quark-gluon plasma (QGP). In these phases the currents of light quarks (vector and axial-vector) can be independently examined for right-handed (RH) and left-handed (LH) quarks. To describe such a quark matter chiral chemical potential can be introduced to quantify the presence of chirality imbalance (ChI) i.e. the difference between the average numbers of RH and LH quarks in the fireball after HIC. In this review talk we will focus our attention on the discussion of the $\mathrm{ChI}$ related developments in heavy ion physics at central collisions and the plans for the future experiments aimed at establishing (or falsifying) the presence of Local spacial Parity Breaking (LPB) in heavy ion data. We describe some of experimental observables in detecting the signal of LPB. A number of measurements is proposed that allow to reach a definite conclusion on the occurrence of LPB effects in non-Abelian QGP produced in central heavy ion collisions and its simulation within a number of QCD-inspired models is outlined. Based on the effective meson theory in the presence of Chern-Simons interaction it is found that the spectrum of massive vector mesons splits into three polarization components with different effective masses. Moreover a resonance broadening occurs that leads to an increase of spectral contribution to the dilepton production as compared to the vacuum state. The asymmetry in production of longitudinally and transversely polarized states of $\rho$ and $\omega$ mesons for various values of the dilepton invariant mass can serve as a characteristic indication of the LPB in CERES, HADES, PHENIX, STAR, NA60 and ALICE experiments.
\end{abstract}

\section{Introduction and Outline}

Nowadays Quantum Chromodynamics (QCD) represents a theory with known symmetries and well established elementary constituents. However their interaction leading to the observed hadron states

\footnotetext{
^e-mail: andrianov@icc.ub.edu

$\star \star$ e-mail: v.andriano@rambler.ru

$\star \star \star$ e-mail: espriu@icc.ub.edu
} 
still needs in the adequate description beyond the perturbation theory, especially, at low and intermediate energies.

It is widely believed that such a non-perturbative dynamics occurs due to the nontrivial topology of QCD vacuum as a consequence of the compactness of non-Abelian color gauge group $S U(3)$. Its compactness allows for topologically nontrivial configurations of the gluon field. These configurations essentially determine the QCD vacuum structure. The latter is composed of the superposition of an infinite set of topologically distinct states connected by tunneling instanton-like transitions [1] and becomes a " $\theta$-vacuum" of the theory [2,3]. It is likely that topological effects in QCD are responsible for the chiral symmetry breaking (see $[4,5]$ for a review).

With the light current quark masses negligible compared to relevant energy scales, the QCD Lagrangian has an approximate chiral symmetry. However this symmetry is not realized in the ground state due to formation of quark-anti-quark pair condensate in the QCD vacuum. This is of course the well-known phenomenon of spontaneously broken chiral symmetry which is a fundamental property of QCD. On the other hand, lattice QCD computations have shown that with increasing temperature such a vacuum condensate will be melting with the chiral symmetry being restored at sufficiently high temperature $T>T_{c} \sim 170 \mathrm{MeV}[6,7]$. Therefore, chiral symmetry restoration at high temperatures is also a basic prediction of QCD. With such a symmetry in light quark sector, the currents (vector and axial-vector) in quark-gluon phase (QGP) can be independently examined for right-handed (RH) and left-handed (LH) quarks. To describe such a matter a chiral chemical potential can be introduced in order that to quantify the presence of chirality imbalance i.e. the difference between the numbers of RH and LH chiral fermions in the system. Quarks with specific chirality have their momentum direction correlated with spin orientation.

There remains however the crucial question of how to achieve a chiral QGP with chirality imbalance in the first place. The process of creating nonzero chirality pertains to the famous "chiral anomaly" $[8,9]$. This anomaly implies the breaking of axial current conservation by quantum loop effects. Correspondingly, locally, chirality imbalance can be generated through nonorthogonal electric and magnetic field strength. Such fields can be either QED EM fields or QCD chromo-EM ones. In QCD the gluon configurations generating chiral imbalance globally exist: they are the topological solitons such as instantons and sphalerons. These topological objects play crucial role for nonperturbative dynamics in QCD vacuum [10] as well as in hot QGP [11, 12]. Such configurations have their global topological winding number $Q_{w}$, and when coupled with chiral fermions, can generate an amount of global chirality imbalance via chiral anomaly: $N_{R}-N_{L}=2 Q_{w}$ for each flavor of light quarks. In short, the chirality imbalance is a direct manifestation of the QCD topological fluctuations and could become observable through the asymmetry in polarizations of dilepton pairs [13] in central heavy ion collisions (HIC) and the Chiral magnetic wave (CMW) in non-central HIC [14]. Summarizing one can say that the Chiral imbalance $(\mathrm{ChI})$ of quarks is a remarkable phenomenon that stems from highly nontrivial interplay of chiral symmetry of QCD, axial anomaly, and the topology of gluon configurations.

In this talk we focus the reader's attention on the discussion of ChI related developments in heavy ion physics at central collisions and the plans for the future experiments aimed at establishing (or falsifying) the presence of Local parity breaking (LPB) in HIC data.

We describe:

- Where and how does chiral imbalance appear: Phenomenology of parity breaking in finite volume.

- Chiral imbalance in quark-gluon phase: topological charge vs. axial (chiral) charge: topological vs. chiral chemical potentials.

- Chiral imbalance in hadronic phase: scalar, pseudoscalar, vector meson spectra[13]. 
- Observables sensitive to chiral imbalance - manifestation of LPB in heavy ion collisions (HIC): dilepton polarization asymmetry [13].

\section{Phenomenology of parity breaking in finite volume}

Parity is a well established global symmetry of strong interactions. Are there any reasons to believe it may be broken in a finite volume?! Local large fluctuations in the topological charge presumably exist in a hot environment and are responsible for LPB.

- Quantum fluctuations of $\theta$ parameter: $P$-odd bubbles [14], their manifestation in Chiral Magnetic Effect (CME)[15]) (an earlier proposal: [16] ).

- New QCD phase characterized by a spontaneous parity breaking due to formation of neutral pionlike background $[17,18]$.

- High energy production of pseudoscalar gluelumps $\Leftrightarrow$ of parity-odd bunches of gluon jets $\Rightarrow$ then a PB background remains inside a hot dense nuclear fireball in HIC !?

Observable effects:

- For peripheral heavy ion collisions they lead to the Chiral Magnetic Effect (CME): Large $\vec{B} \Rightarrow$ large $\vec{E} \Rightarrow$ charge separation[14].

- For central collisions (and light quarks) they correspond to a phase with axial chemical potential $\mu_{5} \neq 0$ located in "fluctons" of few-Fermi size [13].

The two effects are complementary!

\section{Topological charge and topological chemical potential}

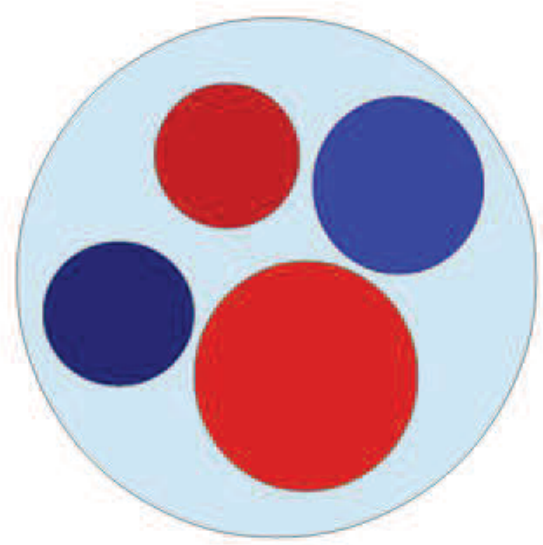

Figure 1. Interplay between domains with positive (in red) and negative (in blue) topological charge in the fireball interior 
In hot QCD (in heavy ion collisions) a metastable topological charge $\left\langle T_{5}\right\rangle$ may arise in a finite volume due to sphaleron-like transitions [19].

$$
T_{5}=\frac{1}{8 \pi^{2}} \int_{\text {vol. }} d^{3} x \varepsilon_{j k l} \operatorname{Tr}\left(G^{j} \partial^{k} G^{l}-i \frac{2}{3} G^{j} G^{k} G^{l}\right)
$$

it may survive for a sizeable lifetime in a heavy-ion fireball

$$
\begin{gathered}
\left\langle\Delta T_{5}\right\rangle \neq 0 \quad \text { for } \quad \Delta t \simeq \tau_{\text {fireball }} \simeq 5 \div 10 \mathrm{fm} / \mathrm{c} \\
(7 \pm 1 \mathrm{fm} / \mathrm{c} \quad[20])
\end{gathered}
$$

For the fireball lifetime one can trigger the value of $\left\langle\Delta T_{5}\right\rangle \neq 0$ introducing into the QCD Lagrangian a topological chemical potential $\mu_{\theta}$ in a gauge invariant way via $\Delta \mathcal{L}_{\text {top }}=\mu_{\theta} \Delta T_{5}$, where

$$
\Delta T_{5}=T_{5}\left(t_{f}\right)-T_{5}(0)=\frac{1}{8 \pi^{2}} \int_{0}^{t_{f}} d t \int_{\text {vol. }} d^{3} x \operatorname{Tr}\left(G^{\mu \nu} \widetilde{G}_{\mu \nu}\right)
$$

We notice that in finite $3+1$ dimensional volume the so-called index theorem does not work and the jump of topological charge is not necessarily integer.

\section{Axial baryon charge and axial chemical potential}

The exact operator law in QCD, the partial conservation of axial current (broken by current quark masses $m_{q}$ for light quarks $q=u, d, s$ and gluon anomaly)

$$
\partial_{\mu} J_{5}^{\mu}-2 i m_{q} J_{5}=\frac{1}{8 \pi^{2}} \operatorname{Tr}\left(G^{\mu v} \widetilde{G}_{\mu v}\right)
$$

predicts the rigid relations between changes in topology of gauge configurations and the induced axial charge (for small quark masses $m_{q} \simeq 0$ ). If the gluon and quark flows across the volume boundary of a fireball are reasonably small (a fireball is metastable) then,

$$
\frac{d}{d t}\left(Q_{5}-2 N_{f} T_{5}\right) \simeq 0, \quad Q_{5}=\int_{\text {vol. }} d^{3} x \sum_{u, d, s} q^{\dagger} \gamma_{5} q=\left\langle N_{L}-N_{R}\right\rangle
$$

and the chiral charge generated in a heavy ion collision is approximately conserved during $\tau_{\text {fireball }}$.

The characteristic left-right oscillation time is governed by inverse quark masses. Indeed if an excess of left(or right)-handed quark states $q_{L}\left(x_{0}=0\right)$ arises in the fireball in the initial moment of central collision then their further evolution in the rest frame can be approximately characterized by the following equation,

$$
q\left(x_{0}\right) \simeq\left(\cos \left(m_{q} x_{0}\right)-i \gamma_{0} \sin \left(m_{q} x_{0}\right)\right) q_{L}(0) .
$$

Therefore after a period $\Delta x_{0} \sim \pi / 2 m_{q}$ left-handed quark states become essentially right-handed.

- For $u, d$ quarks $1 / m_{q} \sim 1 / 5 \mathrm{MeV}^{-1} \sim 40 \mathrm{fm} \gg \tau_{\text {fireball }}$ and the left-right quark mixing can be neglected during fireball life time.

- For $s$ quark $1 / m_{s} \sim 1 / 150 \mathrm{MeV}^{-1} \sim 1 \mathrm{fm} \ll \tau_{\text {fireball }}$ and the chiral charge averaged over the fireball volume and its lifetime vanishes $\left\langle Q_{5}^{s}\right\rangle \simeq 0$ due to left-right oscillations. 
Correspondingly for $u, d$ quarks in a background with the jump of topological charge $\left\langle\Delta T_{5}\right\rangle \neq 0$ or with the chiral (axial) charge $\left\langle Q_{5}\right\rangle \neq 0$ QCD can be equally described at the Lagrangian level by introducing a topological chemical potential $\mu_{\theta}$ or an axial chemical potential $\mu_{5}$

$$
\begin{gathered}
\left\langle\Delta T_{5}\right\rangle \simeq \frac{1}{2 N_{f}}\left\langle Q_{5}\right\rangle \Longleftrightarrow \mu_{5} \simeq \frac{1}{2 N_{f}} \mu_{\theta}, \\
\Delta \mathcal{L}_{\text {top }}=\mu_{\theta} \Delta T_{5} \Longleftrightarrow \Delta \mathcal{L}_{q}=\mu_{5} Q_{5}
\end{gathered}
$$

Thus one can think about a generation of chiral imbalance from non-trivial topological charge or visa versa inducing a topologically non-trivial gluon configuration by imbalance of left- and right-handed quark densities. As both properties are related locally one may get a parity violation generated by either charge in a finite volume of fireball and visible during fireball lifetime.

How does $\mu_{5}$ affect the hadronic phenomenology?

\section{Effective meson theory in a medium with LPB}

In the environment with chiral chemical potential the scalar sector can be described by using the spurion technique in the chiral Lagrangian with an isosinglet $\mu_{5}$ [21],

$$
D_{v} \Longrightarrow D_{v}-i\left\{\mathbf{I}_{q} \mu_{5} \delta_{0 v}, \cdot\right\}=D_{v}-2 i \mathbf{I}_{q} \mu_{5} \delta_{0 v},
$$

where for three light flavors $\mathbf{I}_{q} \equiv \operatorname{diag}[1,1,0]$ in accordance with the preceding discussion. An effective Lagrangian is required to include the lightest isoscalar degrees of freedom such as $\sigma$ and $a_{0}(980)$, which will be mixed with their pseudoscalar partners $\eta, \eta^{\prime}$ and $\pi$, respectively.

In the lowest order of chiral expansion such an effective lagrangian reads,

$$
\begin{gathered}
\mathcal{L}=\frac{1}{4} \operatorname{Tr}\left(D_{\mu} H D^{\mu} H^{\dagger}\right)+\frac{B}{2} \operatorname{Tr}\left[\hat{m}\left(H+H^{\dagger}\right)\right]+\frac{M^{2}}{2} \operatorname{Tr}\left(H H^{\dagger}\right) \\
-\frac{\lambda_{1}}{2} \operatorname{Tr}\left[\left(H H^{\dagger}\right)^{2}\right]-\frac{\lambda_{2}}{4}\left[\operatorname{Tr}\left(H H^{\dagger}\right)\right]^{2}+\frac{c}{2}\left(\operatorname{det} H+\operatorname{det} H^{\dagger}\right) \\
+\frac{d_{1}}{2} \operatorname{Tr}\left[\hat{m}\left(H H^{\dagger} H+H^{\dagger} H H^{\dagger}\right)\right]+\frac{d_{2}}{2} \operatorname{Tr}\left[\hat{m}\left(H+H^{\dagger}\right)\right] \operatorname{Tr}\left(H H^{\dagger}\right)
\end{gathered}
$$

where the $S U(3)_{L} \times S U(3)_{R}$ chirally invariant part is parameterized by constants $M, \lambda_{1,2}, c$ and soft chiral symmetry breaking is realized with the help of vertices containing $\hat{m}=\operatorname{diag}\left[m_{u}, m_{d}, m_{s}\right]$ and constants $B, d_{1,2}$. The convenient splitting of scalar fields is given by

$$
H=\xi \Sigma \xi, \quad \xi=\exp \left(i \frac{\Phi}{2 f}\right), \quad \Phi=\lambda^{a} \phi^{a}, \quad \Sigma=\lambda^{b} \sigma^{b}
$$

The v.e.v. of the neutral scalars are defined as $v_{i}=\left\langle\Sigma_{i i}\right\rangle$ where $i=u, d$, s, and, in the chiral limit $\hat{m}=0$, satisfy the following gap equations:

$$
\begin{gathered}
\left(2 \mu_{5}^{2}+M^{2}\right)-2\left(\lambda_{1}+\lambda_{2}\right) v_{q}^{2}-\lambda_{2} v_{s}^{2}+c v_{s}=0 ; \\
\left(-2 \lambda_{2}+\frac{c}{v_{s}}\right) v_{q}^{2}+M^{2}-\left(2 \lambda_{1}+\lambda_{2}\right) v_{s}^{2}=0,
\end{gathered}
$$

where for non-vanishing isosinglet $\mu_{5}$ we find our solutions to be $v_{u}=v_{d} \equiv v_{q} \neq v_{s}$. 


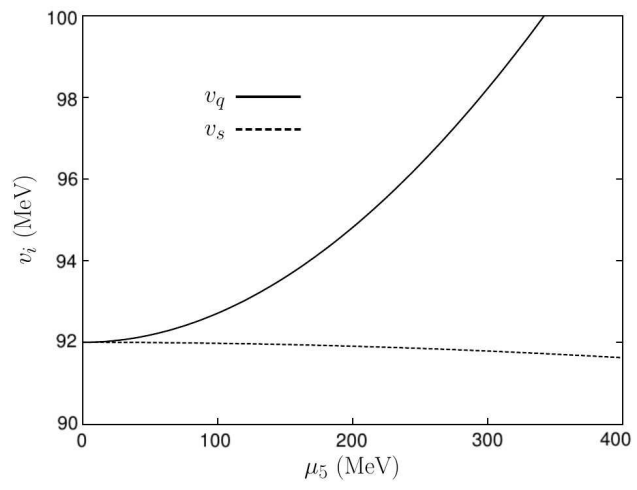

Figure 2. Vacuum condensates in the presence of chiral imbalance

Evidently for large $\mu_{5}$ the correct solution has the following asymptotics,

$$
v_{q}^{2}=\frac{2 \mu_{5}^{2}+M^{2}+\frac{c^{2}}{4 \lambda^{2}}}{2\left(\lambda_{1}+\lambda_{2}\right)}+O\left(1 / \mu_{5}^{2}\right), \quad v_{s}=\frac{c}{2 \lambda_{2}}+O\left(1 / \mu_{5}^{2}\right) .
$$

These solutions are depicted in the Fig.2.

We stress that the consistent solution with a finite limit for strange quark constituent mass $v_{s} \ll v_{q}$ exists if the term breaking $U(1)_{A}$ symmetry is in action. Meantime the constituent masses for light $u, d$ flavours is growing with chiral chemical potential, first as $\sim \mu_{5}^{2}$ and next as $\sim \mu_{5}$. Moreover even if the spontaneous chiral symmetry breaking is absent for $M^{2}<0$ in parity-even environment this CSB will be triggered by chiral imbalance.

The lattice computations perfectly confirm [22] this behavior against chiral chemical potential.

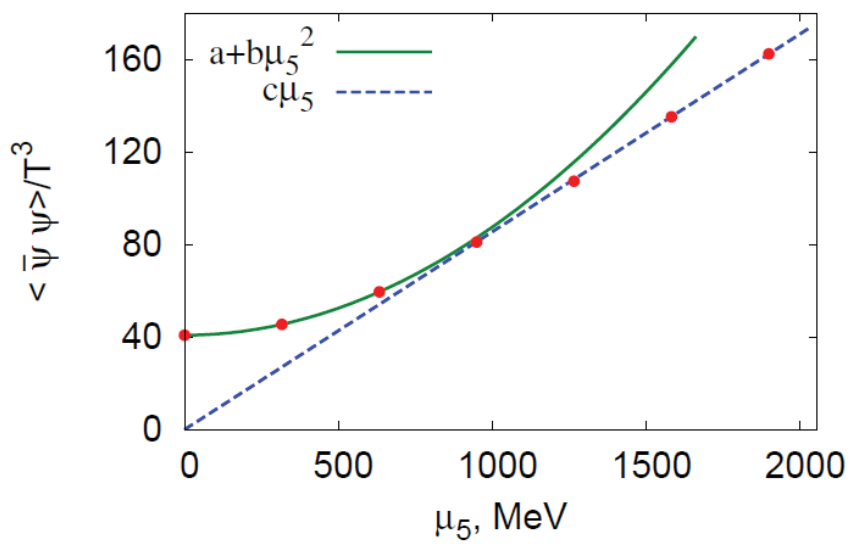

Figure 3. Chiral condensate in two-color QCD lattice as a function of $\mu_{5}$ in the confinement phase $(\mathrm{T}=158$ $\mathrm{MeV})$. 
For further purposes we need the non-strange meson sector and $\eta_{s}$

$$
\begin{gathered}
\Phi=\left(\begin{array}{ccc}
\eta_{q}+\pi^{0} & \sqrt{2} \pi^{+} & 0 \\
\sqrt{2} \pi^{-} & \eta_{q}-\pi^{0} & 0 \\
0 & 0 & \sqrt{2} \eta_{s}
\end{array}\right), \Sigma=\left(\begin{array}{ccc}
v_{u}+\sigma+a_{0}^{0} & \sqrt{2} a_{0}^{+} & 0 \\
\sqrt{2} a_{0}^{-} & v_{d}+\sigma-a_{0}^{0} & 0 \\
0 & 0 & v_{s}
\end{array}\right) \\
\left(\begin{array}{l}
\eta_{q} \\
\eta_{s}
\end{array}\right)=\left(\begin{array}{cc}
\cos \psi & \sin \psi \\
-\sin \psi & \cos \psi
\end{array}\right)\left(\begin{array}{c}
\eta \\
\eta^{\prime}
\end{array}\right)
\end{gathered}
$$

For $\mu_{5}=0$, in the chiral limit $\hat{m}=0$ we adopt $v_{u}=v_{d}=v_{s}=v_{0} \equiv f_{\pi} \approx 92 \mathrm{MeV}$. The coupling constants (in units of $\mathrm{MeV}$ ) are fitted to phenomenology assuming isospin symmetry with the help of $\chi^{2}$ minimization (MINUIT):

$$
\begin{gathered}
b=-3510100 / m, M^{2}=1255600, c=1252.2, \lambda_{1}=67.007, \\
\lambda_{2}=9.3126, d_{1}=-1051.7 / m, d_{2}=523.21 / m,
\end{gathered}
$$

where $m \equiv m_{q}=\left(m_{u}+m_{d}\right) / 2$ and $m / m_{s} \simeq 1 / 25$.

One, of course, should not expect the range of applicability of dim-4 meson lagrangian for chemical potentials larger than few hundreds of $\mathrm{MeV}$. Nevertheless the predictions for lightest states, pions can be well controlled within the chiral perturbation theory.

\subsection{New eigenstates of strong interactions with LPB (isotriplet)}

We present a simple case of mixing due to LPB in the isotriplet sector with $\pi$ and $a_{0}$. The kinetic and mixing terms in the Lagrangian are given by

$$
\mathcal{L}=\frac{1}{2}\left(\partial a_{0}\right)^{2}+\frac{1}{2}(\partial \pi)^{2}-\frac{1}{2} m_{1}^{2} a_{0}^{2}-\frac{1}{2} m_{2}^{2} \pi^{2}-4 \mu_{5} a_{0} \dot{\pi}
$$

where

$$
\begin{aligned}
m_{1}^{2}= & -2\left[M^{2}-2\left(3 \lambda_{1}+\lambda_{2}\right) v_{q}^{2}-\lambda_{2} v_{s}^{2}\right. \\
& \left.-c v_{s}+2\left(3 d_{1}+2 d_{2}\right) m v_{q}+2 d_{2} m_{s} v_{s}+2 \mu_{5}^{2}\right] \\
m_{2}^{2}= & \frac{2 m}{v_{q}}\left[b+\left(d_{1}+2 d_{2}\right) v_{q}^{2}+d_{2} v_{s}^{2}\right]
\end{aligned}
$$

After diagonalization in the momentum representation, the new (momentum-dependent) eigenstates are defined $\tilde{\pi}$ and $\tilde{a}_{0}$.

$\tilde{a}_{0}$ mass shows an enhancement, but $\mu_{5}$ has to be understood as a perturbatively small parameter. A better treatment of $\tilde{a}_{0}$ would require heavier degrees of freedom.

\subsection{New eigenstates of strong interactions with LPB (isosinglet)}

Two new processes are likely to appear inside the fireball: the decays $\eta, \eta^{\prime} \rightarrow \pi \pi$ that are strictly forbidden in QCD on parity grounds.

In a medium where parity is broken: are these processes relevant within the fireball? Can these particles reach thermal equilibrium?!

There are no $O\left(p^{2}\right)$ terms in the chiral Lagrangian involving vertices $\eta \pi \pi$. 

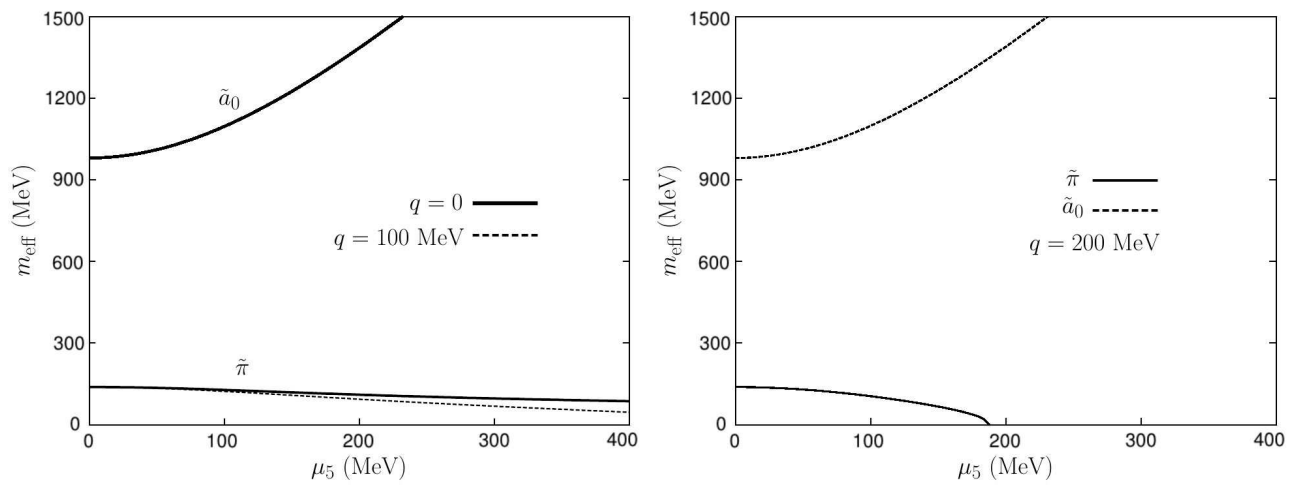

Figure 4. For high energies $k_{0},|\vec{k}|>m_{1} m_{2} /\left(4 \mu_{5}\right) \equiv k_{\tilde{\pi}}^{c}$, in-medium $\tilde{\pi}$ goes tachyonic. Nevertheless, energies are always positive (no vacuum instabilities).

$O\left(p^{4}\right)$ terms lead to couplings such as

$$
\mathcal{L}_{\eta \pi \pi} \sim \frac{16 \mu_{5}}{F_{\eta} f_{\pi}^{2}} L \partial \eta \partial \pi \partial \pi
$$

where $L \sim 10^{-3}$. A rough estimate of the partial width shows a strong dependence on $\mu_{5}$ as $\Gamma_{\eta \rightarrow \pi \pi} \propto \mu_{5}^{2}$ and gives values similar or higher than $\Gamma_{\rho \rightarrow \pi \pi}=150 \mathrm{MeV}$.

In the isosinglet sector, we show the mixing of $\eta, \sigma$ and $\eta^{\prime}$. The kinetic and mixing terms in the Lagrangian are given by

$$
\begin{aligned}
\mathcal{L}= & \frac{1}{2}\left[(\partial \sigma)^{2}+\left(\partial \eta_{q}\right)^{2}+\left(\partial \eta_{s}\right)^{2}\right]-\frac{1}{2} m_{3}^{2} \sigma^{2}-\frac{1}{2} m_{4}^{2} \eta_{q}^{2}-\frac{1}{2} m_{5}^{2} \eta_{s}^{2} \\
& -4 \mu_{5} \sigma \dot{\eta}_{q}-2 \sqrt{2} c v_{q} \eta_{q} \eta_{s},
\end{aligned}
$$

where

$$
\begin{aligned}
m_{3}^{2}= & -2\left(M^{2}-6\left(\lambda_{1}+\lambda_{2}\right) v_{q}^{2}-\lambda_{2} v_{s}^{2}+c v_{s}\right. \\
& \left.+6\left(d_{1}+2 d_{2}\right) m v_{q}+2 d_{2} m_{s} v_{s}+2 \mu_{5}^{2}\right), \\
m_{4}^{2}= & \frac{2 m}{v_{q}}\left[b+\left(d_{1}+2 d_{2}\right) v_{q}^{2}+d_{2} v_{s}^{2}\right]+2 c v_{s}, \\
m_{5}^{2}= & \frac{2 m_{s}}{v_{s}}\left[b+2 d_{2} v_{q}^{2}+\left(d_{1}+d_{2}\right) v_{s}^{2}\right]+\frac{c v_{q}^{2}}{v_{s}} .
\end{aligned}
$$

After diagonalization, the new eigenstates are $\tilde{\sigma}, \tilde{\eta}$ and $\tilde{\eta}^{\prime}$.

Again, for high energies $k_{0},|\vec{k}|>k_{\tilde{\eta}}^{c}$ with $k_{\tilde{\eta}}^{c} \equiv \frac{m_{3}}{4 \mu_{5} m_{5}} \sqrt{m_{4}^{2} m_{5}^{2}-8 c^{2} v_{q}^{2}}$, in-medium $\tilde{\eta}$ goes tachyonic. $\tilde{\eta}^{\prime}$ mass also shows an enhancement and a better treatment would require the inclusion of heavier degrees of freedom. 

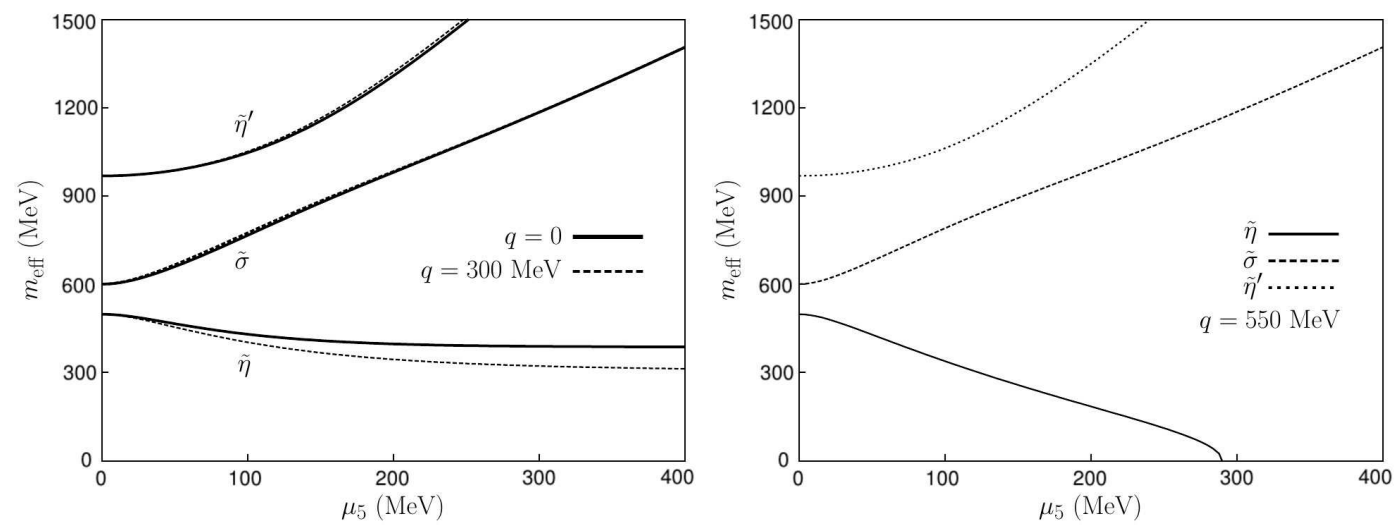

The cubic couplings used to calculate the widths $\tilde{\eta}, \tilde{\sigma}, \tilde{\eta}^{\prime} \rightarrow \tilde{\pi} \tilde{\pi}$ from the Lagrangian are given by

$$
\begin{aligned}
& \mathcal{L}_{\sigma a a}=2\left[\left(3 d_{1}+2 d_{2}\right) m-2\left(3 \lambda_{1}+\lambda_{2}\right) v_{q}\right] \sigma \vec{a}_{0}^{2}, \\
& \mathcal{L}_{\sigma \pi \pi}=\frac{1}{v_{q}^{2}}\left[(\partial \vec{\pi})^{2} v_{q}-\left(b+3\left(d_{1}+2 d_{2}\right) v_{q}^{2}+d_{2} v_{s}^{2}\right) m \vec{\pi}^{2}\right] \sigma, \\
& \mathcal{L}_{\eta a \pi}=\frac{2}{v_{q}^{2}} \vec{a}_{0}\left[\partial \eta_{q} \partial \vec{\pi} v_{q}-\left(b+\left(3 d_{1}+2 d_{2}\right) v_{q}^{2}+d_{2} v_{s}^{2}\right) m \eta_{q} \vec{\pi}\right], \\
& \mathcal{L}_{\sigma a \pi}=-\frac{4 \mu_{5}}{v_{q}} \sigma \overrightarrow{a_{0}} \dot{\vec{\pi}}, \quad \mathcal{L}_{\eta a a}=-\frac{2 \mu_{5}}{v_{q}} \dot{\eta}_{q} \vec{a}_{0}^{2}, \quad \mathcal{L}_{\eta \pi \pi}=0 .
\end{aligned}
$$

After diagonalization, one replaces the initial $\left\{\eta_{q}, \eta_{s}, \sigma\right\}$ to $\left\{\tilde{\eta}, \tilde{\sigma}, \tilde{\eta}^{\prime}\right\}$ and $\left\{\pi, a_{0}\right\}$ to $\left\{\tilde{\pi}, \tilde{a}_{0}\right\}$.

The widths are firstly computed at the rest frame of the decaying particle and secondly with a boosted particle.

$\tilde{\eta}$ exhibits a smooth behaviour with $\left\langle\Gamma_{\tilde{\eta}}\right\rangle \sim 60 \mathrm{MeV} \leftrightarrow$ mean free path $\sim 3 \mathrm{fm} \lesssim L_{\text {fireball }} \sim 5 \div 10$ fm. Possible thermalization! Down to $\mu_{5} \sim 100 \mathrm{MeV}, \tilde{\sigma}$ width decreases and becomes stable. The visible bumps in these two channels seem to reflect the tachyonic nature of the decaying $\tilde{\pi}$. $\tilde{\eta}^{\prime}$ width grows up to the $\mathrm{GeV}$ scale (violation of unitarity). More degrees of freedom are needed.
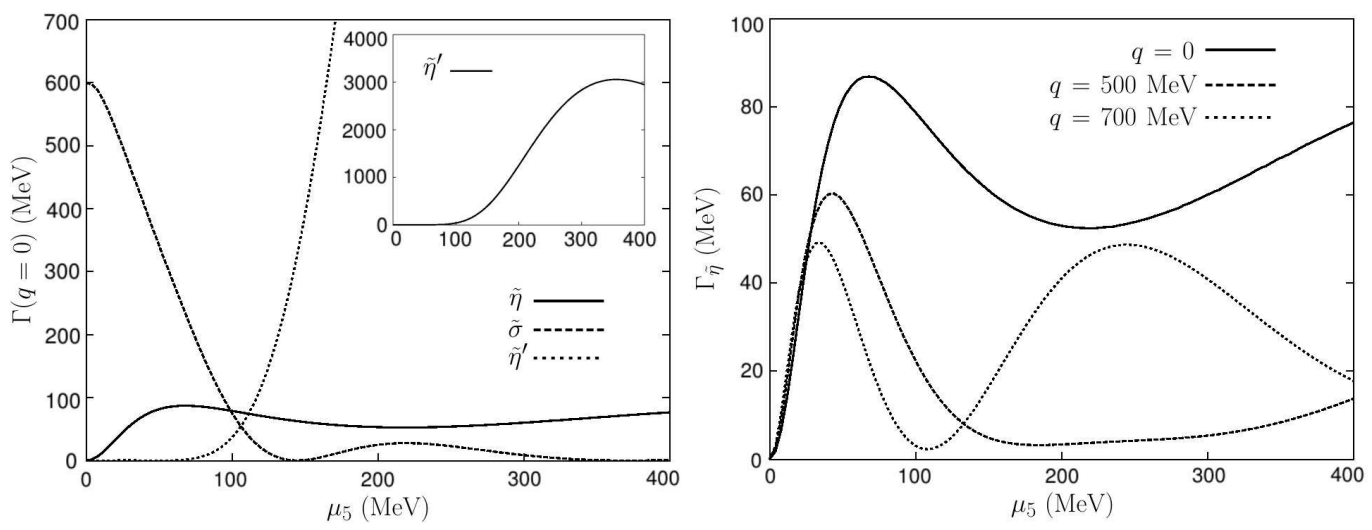

Small variations at low 3-momenta: 2 initial bumps slowly separate as one increases $q . \Gamma_{\tilde{\eta}}\left(\mu_{5}, q\right)$ exhibits a saddle point at $\mu_{5}^{*} \sim 240 \mathrm{MeV}$ and $q^{*} \sim 500 \mathrm{MeV}$. For large 3-momenta, a third interme- 
diate bump appears (creation of 2 tachyons) and grows fast as one increases $q$ becoming the global maximum when $q \gtrsim 700 \mathrm{MeV}$.

\section{Vector mesons in a medium with LPB}

LPB can be investigated in e.m. production of leptons and photons in hot/dense nuclear matter via heavy ion collisions.

- Thus e.m. interaction extends the axial charge by e.m. anomaly,

$$
Q_{5}^{q} \rightarrow \tilde{Q}_{5}=Q_{5}^{q}-T_{5}^{\mathrm{em}}, \quad T_{5}^{\mathrm{em}}=\frac{N_{c}}{8 \pi^{2}} \int_{\text {vol. }} d^{3} x \varepsilon_{j k l} \operatorname{Tr}\left(\hat{A}^{j} \partial^{k} \hat{A}^{l}\right)
$$

- Now chiral chemical potential $\mu_{5}$ is conjugated to (nearly) conserved $\tilde{Q}_{5}$.

- In the hadron phase QCD bosonization of total $\tilde{Q}_{5}$ can be realized [13] with the help of meson Lagrangian and the Vector Meson Dominance providing a good precision in coefficients.

$$
\begin{gathered}
\mathcal{L}_{\text {int }}=\bar{q} \gamma_{\mu} \hat{V}^{\mu} q ; \quad \hat{V}_{\mu} \equiv-e A_{\mu} Q+\frac{1}{2} g_{\omega} \omega_{\mu} \mathbb{I}+\frac{1}{2} g_{\rho} \rho_{\mu}^{0} \tau_{3}, \\
\left(V_{\mu, a}\right) \equiv\left(A_{\mu}, \omega_{\mu}, \rho_{\mu}^{0}\right)
\end{gathered}
$$

where $Q=\frac{\tau_{3}}{2}+\frac{1}{6}, g_{\omega} \simeq g_{\rho} \equiv g \simeq 6$.

In the parity-odd effective lagrangian for vector mesons the following term is generated

$$
\Delta \mathcal{L} \simeq \varepsilon^{\mu \nu \rho \sigma} \operatorname{Tr}\left[\zeta_{\mu} V_{\nu} V_{\rho \sigma}\right]
$$

with $\zeta_{\mu}=\zeta \delta_{\mu 0}$ for a spatially homogeneous and isotropic background and $\zeta \propto \mu_{5}$.

Two different cases of isospin structure for $\mu_{5}$ :

- Isosinglet pseudoscalar background $(T \gg \mu)$ [RHIC, LHC]

- Pion-like (isotriplet) background $(\mu \gg T)$ [FAIR, NICA]

Massive MCS electrodynamics for vector mesons is described by the following lagrangian

$$
\mathcal{L}_{M C S}=-\frac{1}{4} F^{\alpha \beta}(x) F_{\alpha \beta}(x)+\frac{1}{2} m^{2} A_{v}(x) A^{v}(x)+\frac{1}{2} \zeta_{\mu} A_{v}(x) \tilde{F}^{\mu v}(x)+\text { g.f. }
$$

In momentum space wave Eqs.

$$
\left[g^{\lambda v}\left(k^{2}-m^{2}\right)-k^{\lambda} k^{v}+i \varepsilon^{\lambda \nu \alpha \beta} \zeta_{\alpha} k_{\beta}\right] \mathbf{a}_{\lambda}(k)=0 ; \quad k^{\lambda} \mathbf{a}_{\lambda}(k)=0 .
$$

Energy spectrum for transversal polarizations is governed by

$$
\omega_{\mathbf{k}, \pm}=\sqrt{\mathbf{k}^{2}+m^{2} \pm \zeta|\mathbf{k}|},
$$

and for longitudinal polarization it remains intact,

$$
\omega_{\mathbf{k}, L}=\sqrt{\mathbf{k}^{2}+m^{2}}
$$

After diagonalization of mass matrix the mass spectrum is given by

$$
m_{V, \epsilon}^{2}=m_{V}^{2}-\epsilon \zeta|\vec{k}|
$$


where $\epsilon=0, \pm 1$ is the type of meson polarization. Thus $m_{V,+}^{2}<m_{V, L}^{2}<m_{V,-}^{2}$.

If $\hat{\zeta}$ is an isosinglet the photon itself happens to be unaffected by CS interaction.

The position of the poles for \pm polarized vector mesons is moving with wave vector $|\vec{k}|$.

This splitting unambiguously signifies LPB. Can it be measured?

$\rightarrow$ Dilepton production in HIC from the decays $\rho, \omega \rightarrow e^{+} e^{-}$potentially contains sufficient information on LPB.

\subsection{Manifestation of LPB in heavy ion collisions in dilepton production}

$L, \pm$ contribution for vector mesons before acceptance corrections:

$$
\begin{aligned}
& \frac{d N_{e e}^{\epsilon}}{d M} \simeq c_{V} \frac{\alpha^{2} \Gamma_{V} m_{V}^{2}}{3 \pi^{2} g^{2} M^{2}}\left(\frac{M^{2}-n_{V}^{2} m_{\pi}^{2}}{m_{V}^{2}-n_{V}^{2} m_{\pi}^{2}}\right)^{3 / 2} \\
& \times \sum_{\epsilon} \int_{M}^{\infty} d k_{0} \frac{\sqrt{k_{0}^{2}-M^{2}}}{e^{k_{0} / T}-1} \frac{m_{V, \epsilon}^{4}}{\left(M^{2}-m_{V, \epsilon}^{2}\right)^{2}+m_{V, \epsilon}^{4} \frac{\Gamma_{V}^{2}}{m_{V}^{2}}},
\end{aligned}
$$

where $n_{V}=2,0 ;|\vec{k}|=\sqrt{k_{0}^{2}-M^{2}}$ and $M^{2}>n_{V}^{2} m_{\pi}^{2} \cdot c_{V}$ absorbs combinatorial factors different for $\rho$ and $\omega, \mu$, finite volume suppression.

Simulations of distorted spectral functions are implemented with PHENIX acceptance: $\left|y_{e e}\right|<$ $0.35,\left|\vec{p}_{t}^{e}\right|>200 \mathrm{MeV}$, gaussian $M_{e e}$ smearing (width=10 MeV)
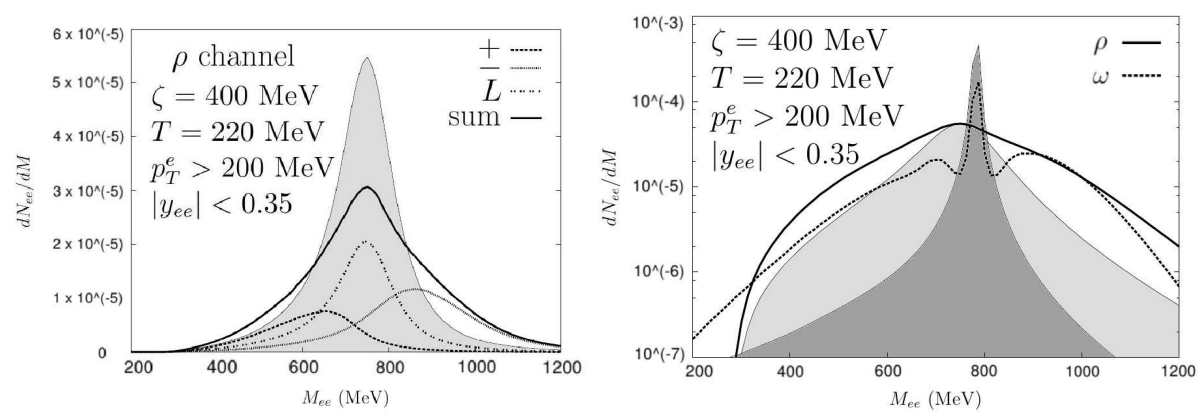

Figure 5. On the left: polarization splitting in $\rho$ spectral function for LPB $\zeta=400 \mathrm{MeV}\left(\mu_{5}=290 \mathrm{MeV}\right)$ compared with $\zeta=0$ (shaded region). On the right: in-medium $\rho$ and $\omega$ channels (solid and dashed line) and their vacuum contributions (light and dark shaded regions) for $\zeta=400 \mathrm{MeV}$. In-medium $\rho$ is enhanced by a factor 1.8 due to $\pi \pi$ regeneration into $\rho$. 
The PHENIX/STAR anomaly: an abnormal $e^{+} e^{-}$yield is observed in central HIC.
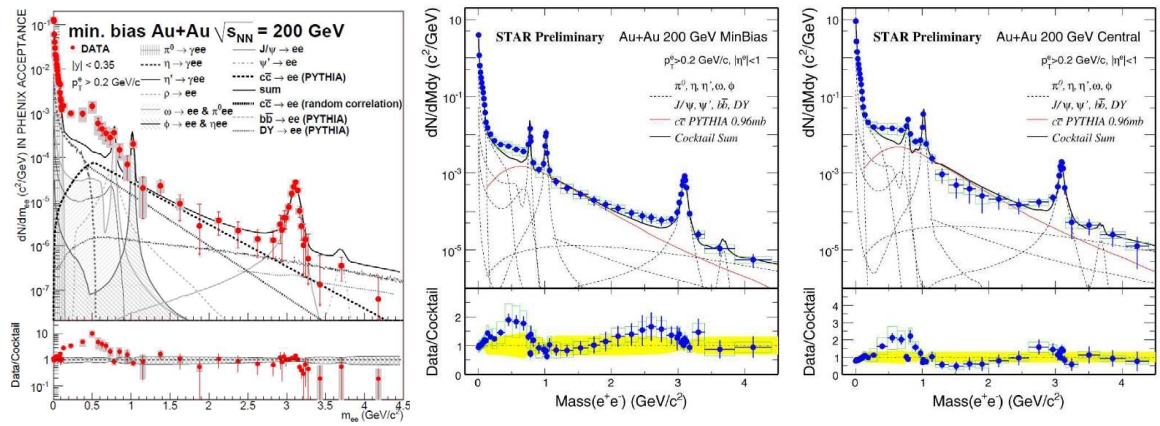

Figure 6. Abnormal excess of dileptons: on the left PHENIX data[23] and on the right STAR data[24]

It may be explained partially by the local parity breaking shown in Fig. 5

\subsection{Polarization asymmetry: dilepton angular distributions}

In [25] it was suggested to detect a splitting of dilepton polarizations averaged over angular sectors. There are two convenient choices of this splitting.

Case A: the angle $\theta_{A}$ between the two outgoing leptons in the laboratory frame.
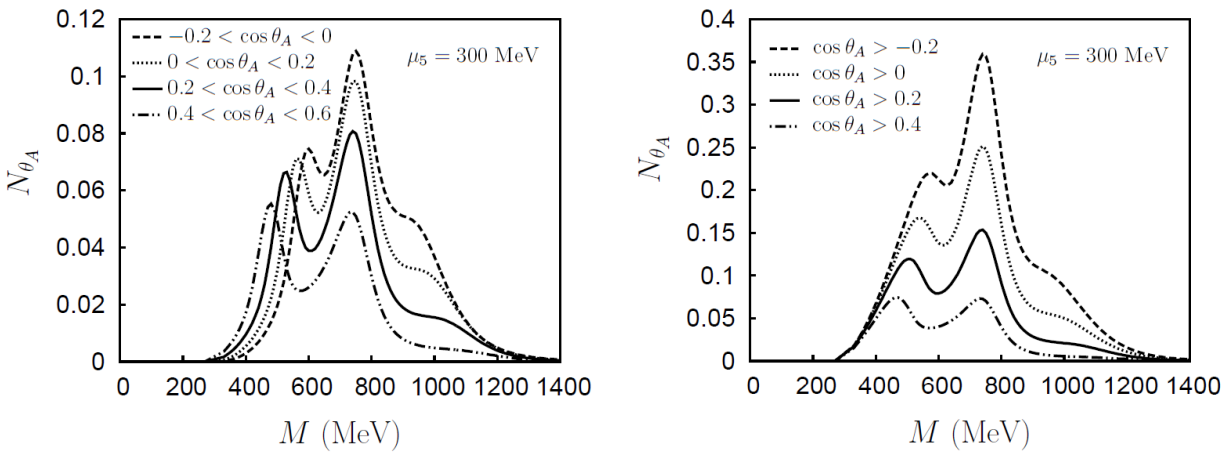

Figure 7. The $\rho$ spectral function is presented for fixed $\mu_{5}=300 \mathrm{MeV}$. $\cos \theta_{A} \in$ $[-0.2,0],[0,0.2],[0.2,0.4],[0.4,0.6]$ and $[0.6,0.8]$ in the left panel, and $\cos \theta_{A} \geq-0.2,0,0.2,0.4$ in the right one. Results are corrected for PHENIX experimental acceptance.

Case B: the angle $\theta_{B}$ between one of the two outgoing leptons in the laboratory frame and the same lepton in the dilepton rest frame 

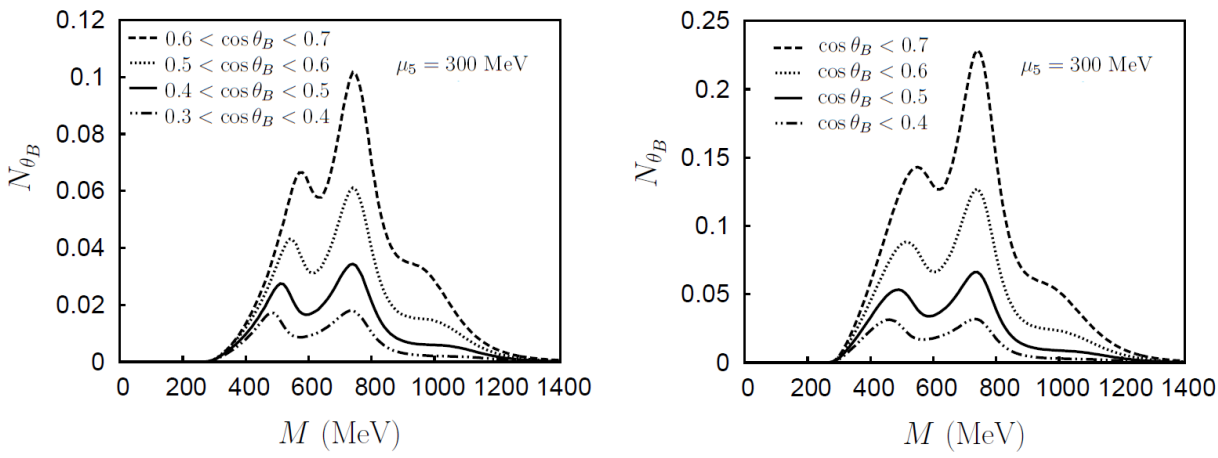

Figure 8. The $\rho$ spectral function is presented for fixed $\mu_{5}=300 \mathrm{MeV} \cdot \cos \theta_{B} \in[0.3,0.4],[0.4,0.5],[0.5,0.6]$ and $[0.6,0.7]$ in the left panel, and $\cos \theta_{B} \leq 0.4,0.5,0.6,0.7$ in the right one. Results are corrected for PHENIX experimental acceptance.

\section{Conclusions and Outlook}

In the report a medium (a fireball) is regarded with chiral imbalance occurring in heavy ion collisions at high energies. To describe such a quark (hadron) matter chiral chemical potential can be introduced to quantify the presence of chirality imbalance i.e. the difference between the numbers of Right-(Left-)handed quarks in the fireball after HIC at central collisions. In such a medium (in hadron phase) the possibility of phase formation with a Local special Parity Breaking is described. Based on the effective meson theory in the presence of Chern-Simons interaction it is found that the spectrum of massive vector mesons splits into three polarization components with different effective masses. Accordingly a resonance broadening occurs that leads to an increase of spectral contribution to the dilepton production as compared to the vacuum state. In particular, in a hadron phase some of experimental observables are presented for detecting the signal of LPB. The asymmetry in production of longitudinally and transversely polarized states of $\rho$ and $\omega$ mesons for various values of the dilepton invariant mass can serve as a characteristic indication of the LPB in CERES, HADES, PHENIX, STAR, NA60 and ALICE experiments. Also QCD-motivated models are considered from whose properties the phenomenon of LPB could be found. The results are compared with lattice calculations for the investigated phases.

\section{Acknowledgements}

This work has been supported through grants FPA2013-46570, 2014-SGR-104 and Consolider CPAN. Funding was also partially provided by the Spanish MINECO under project MDM-2014-0369 of ICCUB (Unidad de Excelencia 'Maria de Maeztu'). A.A. and V.A. were supported by Grant RFBR projects 16-02-00348 and also got a financial support of SPbSU, in particular, by projects 11.42.1049.2016, 11.42.1051.2016.

\section{References}

[1] A.A. Belavin, A.M. Polyakov, A.S. Schwartz and Y. S. Tyupkin, Phys. Lett. B 59, 85 (1975).

[2] C. G. Callan, Jr., R. F. Dashen and D. J. Gross, Phys. Lett. B 63, 334 (1976). 
[3] G. 't Hooft, Phys. Rev. D 14, 3432 (1976) [Erratum-ibid. D 18,2199 (1978)].

[4] D.I. Dyakonov and V.Y. Petrov, Phys. Lett. B 147, 351 (1984); Sov. Phys. JETP 62, 204 (1985); ibid. 62, 431 (1985); Nucl. Phys. B 272, 457 (1986).

[5] T. Schaefer and E. V. Shuryak, Rev. Mod. Phys. 70, 323 (1998); [hep-ph/9610451]

[6] S. Borsanyi et al. [Wuppertal-Budapest Collaboration], JHEP. 073, 1009, (2010).

[7] A. Bazavov et al., Phys. Rev. D 85, 054503 (2012).

[8] S. L. Adler, Phys. Rev. 177, 2426 (1969).

[9] J. S. Bell and R. Jackiw, Nuovo Cim. A 60, 47 (1969).

[10] G. 't Hooft, hep-th/0010225; T. Schaefer and E. V. Shuryak, Rev. Mod. Phys. 70, 323 (1998).

[11] E. Shuryak, arXiv:1412.8393 [hep-ph]; Prog. Part. Nucl. Phys. 62, 48 (2009).

[12] J. Xu, J. Liao and M. Gyulassy, Chin. Phys. Lett. 32, no. 9, 092501 (2015); arXiv:1508.00552 [hep-ph].

[13] A.A. Andrianov, V.A. Andrianov, D. Espriu, X. Planells, Physics Letters B 710,230 (2012); Phys. Rev. D 90, 034024 (2014).

[14] D.E. Kharzeev, R.D. Pisarski, M.H.G. Tytgat, Phys. Rev. Lett. 81, 512 (1998); K. Buckley, T. Fugleberg, and A. Zhitnitsky, Phys. Rev. Lett. 84, 4814 (2000); D.E. Kharzeev, Phys. Lett. B 633, 260 (2006); D.E. Kharzeev, Ann. Phys. (NY) 325, 205 (2010); D.E. Kharzeev, A. Zhitnitsky, Nucl. Phys. A 797, 67 (2007); D.E. Kharzeev, L.D. McLerran, H.J. Warringa, Nucl. Phys. A 803, 227 (2008); K. Fukushima, D.E. Kharzeev, H.J. Warringa, Phys. Rev. D 78, 074033 (2008); K. Fukushima, D.E. Kharzeev, H.J. Warringa, Nucl. Phys. A 836, 311 (2010).

[15] T. D. Lee and G. C. Wick Phys. Rev. D 9, 2291 (1974).

[16] A.Vilenkin, Phys.Rev. D 22, 3080 (1980).

[17] A.A.Anselm, Phys.Lett. B 217,169 (1989); A.A.Anselm, M.G.Ryskin, A.G.Shuvaev, Surv. in HEP 11, 27 (1997.

[18] A.A. Andrianov, D. Espriu, Phys. Lett. B 663,450 (2008); A.A. Andrianov, V.A. Andrianov, D. Espriu, Phys. Lett. B 678,416 (2009).

[19] F. R. Klinkhamer and N. S. Manton, Phys. Rev. D 30, 2212 (1984); V. A. Kuzmin, V. A. Rubakov, and M. E. Shaposhnikov, Phys. Lett. B 155, 36 (1985); L. McLerran, E. Mottola, and M. E. Shaposhnikov, Phys. Rev. D 43, 2027 (1991); G. D. Moore and K. Rummukainen, Phys. Rev. D 61, 105008 (2000); E. Shuryak and I. Zahed, Phys. Rev. D 67, 014006 (2003).

[20] R. Rapp and J. Wambach "Chiral symmetry restoration and dileptons in relativistic heavy-ion collisions," in: Advances in Nuclear Physics (Adv. Phys. Part. Nucl. (APPN), Vol. 25, J. W. Negele and E. Vogt, eds.), Kluwer, New York (2002), pp. 1-205; W. Liu and R. Rapp, Nucl. Phys. A 796, 101 (2007); H. van Hees and R. Rapp, Nucl. Phys. A 806, 339 (2008).

[21] A. A. Andrianov, D. Espriu \& X. Planells, Eur. Phys. J. C 73:2294 (2013).

[22] V. V. Braguta and A. Y. Kotov, Phys. Rev. D 93, no. 10, 105025 (2016); V. V. Braguta, V. A. Goy, E. M. Ilgenfritz, A. Yu. Kotov, A. V. Molochkov, M. Muller-Preussker, and B. Petersson. JHEP, 06:094, 2015.

[23] A. Adare et al. [PHENIX Collaboration], Phys. Rev. C 81 (2010) 034911, arXiv:0912.0244 [nucl-ex].

[24] L. Adamczyk et al. [STAR Collaboration], Phys. Rev. Lett. 113, no. 2, 022301 (2014) Addendum: [Phys. Rev. Lett. 113, no. 4, 049903 (2014)] arXiv:1312.7397 [hep-ex].

[25] A.A. Andrianov, V.A. Andrianov, D. Espriu, X. Planells, Phys. Rev. D 90, 034024 (2014). 